\title{
A planning problem combining calculus of variations and optimal transport
}

\author{
G. Carlier, A. Lachapelle*
}

March 29, 2010

\begin{abstract}
We consider some variants of the classical optimal transport where not only one optimizes over couplings between some variables $x$ and $y$ but also over some control variables governing the evolutions of these variables with time. Such a situation is motivated by an assignment problem of tasks with workers whose characteristics can evolve with time (and be controlled). We distinguish between the coupled and decoupled case. The coupled case is a standard optimal transport with the value of some optimal control problem as cost. The decoupled case is more involved since it is nonlinear in the transport plan.
\end{abstract}

Keywords: optimal transport, optimal control.

\section{Introduction}

In the classical optimal transport problem, a planner has to find some coupling or transport plan between two nonnegative measures of equal total mass (say 1) so as to minimize average cost. The Monge-Kantorovich problem is then the linear program consisting in finding a cost minimizing measure having prescribed marginals on variables, say $x$ and $y$. Such problems have been very much studied in recent years (see [11], [12])... Interestingly, transport/assignment problems have their modern roots in planning problems (optimally transporting coal from mines to steel factories for instance) and the pioneering works of Kantorovich in the USSR and Koopmans in the West were awarded by the Nobel prize in economics in 1975. Typical

${ }^{*}$ CEREMADE, UMR CNRS 7534, Université Paris IX Dauphine, Pl. de Lattre de Tassigny, 75775 Paris Cedex 16, FRANCE carlier@ceremade.dauphine.fr, lachapelle@ceremade.dauphine.fr 
applications are optimal allocation of resources or assignment problems; for other economic applications, we refer to the recent work of Ekeland [4], [5] and Chiappori, McCann and Nesheim [3] relating matching problems and hedonic equilibria to optimal transport theory.

If, to fix ideas, we think of a firm having to assign $N$ workers of different skills $x$ to $N$ tasks of different difficulties $y$, the assignment problem simply consists in assigning a task to each worker (for instance assigning most difficult tasks to most skilled workers). It is however reasonable to think that, in addition to designing such an assignment, the firm has the possibility to act directly on the workers' types using some (costly) control variable like internal training. The same of course applies to the task type $y$ that may change with time if, for example, some investments are made to make the task easier or quicker to execute. This is where optimal control comes into play. The problems we consider in the present paper are precisely intended to deal with such situations where a planner or firm has at disposal as decision variables not only the initial assignment but also control variables that affect the dynamics of the state variables $x$ and $y$. More precisely, we consider the case where the planner chooses the initial coupling between $x$ and $y$, initially distributed according to respective probabilities $\mu_{0}$ and $\nu_{0}$, and a control variable governing the evolutions of the characteristics $x$ and $y$. It is assumed that a pair (worker/task) that is initially formed at time 0 with initial characteristics $(x, y)$ will remain paired or married during a fixed period although its characteristics may evolve with time, this captures the idea that for instance workers are assigned a certain task during some minimal period.

We will consider two different cases that we respectively call the coupled and the decoupled case and will compare them. The coupled problem thus amounts to solve a standard optimal transport where the cost function simply is the value function of some optimal control problem. In the decoupled case, we require in addition that the control variable for $x$ has to be chosen independently of $y$ (for instance the planner offers the same training to all the workers of same skill, independently of the task they will be assigned or he invests). This case is more involved since it is not linear (and not even lower semicontinuous) in the transport plan. Our main result is a relaxation result stating that, under quite general assumptions, the coupled and decoupled problems have the same value but the latter may fail to have solutions (see section 3). 


\section{Coupled vs decoupled case}

\subsection{The coupled problem}

We consider a period $[0, T]$, a population of workers whose characteristics (productivity or skills, say) are captured by a vector $X \in \mathbb{R}^{d}$, and evolve with time. We consider also a certain distribution of tasks characteristics that for simplicity, we assume to be fixed during the period. Again for simplicity, we assume that the dynamics for the state variable $X$ simply is $\dot{X}=u$ where $u$ is a control chosen by the firm to improve its employees skills. The running cost is given by some continuous Lagrangian $(x, u, y) \in \mathbb{R}^{3 d} \mapsto L(x, u, y)$ that is convex with $q$-growth in the variable $u$ for some $q>1$, and the terminal cost is $(x, y) \in \mathbb{R}^{2 d} \mapsto \phi(x, y)$. The value function is then defined for every $(x, y) \in \mathbb{R}^{d} \times \mathbb{R}^{d}$ by

$$
v(x, y):=\inf _{X \in W^{1, q}, X(0)=x} \int_{0}^{T} L(X(t), \dot{X}(t), y) d t+\phi(X(T), y) .
$$

In addition to optimally train its employees, the firm has to decide at the beginning how to match workers skills to tasks difficulties. We assume that the distribution of workers' types at $t=0$ is given by some Borel probability measure $\mu_{0}$ and that the distribution of tasks is given by a Borel probability measure $\nu_{0}$. The initial distribution $\gamma$ for the pairing betwen employees and tasks is chosen by the controller, it is a coupling between $\mu_{0}$ and $\nu_{0}$ and thus belongs to the set of transport plan $\Pi\left(\mu_{0}, \nu_{0}\right)$ consisting of all Borel probability measures $\gamma$ on $\mathbb{R}^{d} \times \mathbb{R}^{d}$ having $\mu_{0}$ and $\nu_{0}$ as marginals. For the sake of simplicity, we also assume tat both $\mu_{0}$ and $\nu_{0}$ are compactly supported on $\mathbb{R}^{d}$. The optimal pairing is then found by solving the mass transportation problem

$$
(\mathcal{P}) \inf _{\gamma \in \Pi\left(\mu_{0}, \nu_{0}\right)} I(\gamma)=\int_{\mathbb{R}^{d} \times \mathbb{R}^{d}} v(x, y) d \gamma(x, y)
$$

where $v$ is given by $(2.1)$. It is well-known that $(\mathcal{P})$ admits solutions as soon as $v$ is lower semicontinuous.

There are also very natural cases where there is uniqueness of an optimal pairing that is in fact given by a transport map (that is the optimal pairing between $x$ and $y$ is of the deterministic form $x \mapsto S(x)$ ). Indeed, consider the case where the running and terminal costs respectively take the form

$$
c(x-y)+F(u), \text { and } \phi(x-y)
$$

where $c, \phi$ and $F$ are bounded from below, smooth and strictly convex functions, with $\Lambda$ id $\geq D^{2} F \geq \lambda$ id for some $\Lambda>0, \lambda>0$. In this case the value 
function is given by

$$
v(x, y)=\inf _{X \in H^{1}, X(0)=x} \int_{0}^{T}(c(X(t)-y)+F(\dot{X}(t))) d t+\phi(X(T)-y) .
$$

We then rewrite the value as $v(x, y)=w(x-y)$ where

$$
w(z)=\inf _{X \in H^{1}, X(0)=z} \int_{0}^{T}(c(X(t))+F(\dot{X}(t))) d t+\phi(X(T)) .
$$

It is now easy to check that $w$ is a $C^{1}$ and strictly convex function. Then, it is well-known (see [8], [7]) that for smooth and strictly convex costs of $x-y$, the corresponding optimal transport problem possesses a unique solution that is given by an optimal transport map $S$ as soon as $\mu_{0}$ is absolutely continuous with respect to the Lebesgue measure (see [8]).

Remark 2.1. One could also consider more general controlled stochastic dynamics

$$
d Z_{t}=b\left(Z_{t}, u_{t}\right) d t+\sigma\left(Z_{t}, u_{t}\right) d W_{t}, t \in[0, T]
$$

for the joint evolution of the state variable $Z:=(X, Y)$. For a given class $\mathcal{U}$ of admissible controls, and to each initial condition $(x, y)$ is associated the minimal cost

$$
v(x, y):=\inf _{u \in \mathcal{U}} \mathbb{E}\left(\int_{0}^{T} c\left(Z_{s}^{x, y, u}, u_{s}\right) d s+\phi\left(Z_{T}^{x, y, u}\right)\right) .
$$

Under standard regularity and polynomial growth conditions, it is well known that $v$ is continuous with polynomial growth (see [6]). Then the coupled problem consists again in finding a transport plan $\gamma$ between $\mu_{0}$ and $\nu_{0}$ that minimizes the quantity $\int v(x, y) d \gamma(x, y)$ and the existence of an optimal transport plan can be obtained by standard arguments as soon as $\mu_{0}$ and $\nu_{0}$ possess suitable moments.

\subsection{The decoupled probem}

In the coupled problem $(\mathcal{P})$ considered previously, the situation is rather simple since the problem amounts first to compute the value function and then to solve the optimal transport problem where the cost is precisely this value function. However, this requires that the optimizer is able to design a control that depends on both state variables $x$ and $y$. If one thinks of $x$ as being workers' skills and $y$ the difficulty of some task it may be reasonable to assume in certain cases that the control governing the evolution of $x$ (training, 
education...) is the same for every worker of type $x$ independently of the task $y$ he will be assigned.

Of course, $(\mathcal{P})$ possessses solutions as soon as $v$ is continuous (or more generally lower semicontinuous). In the decoupled case, $X$ is not allowed to depend on $y$ but has instead to minimize the conditional average of the cost. By the disintegration theorem, every $\gamma \in \Pi\left(\mu_{0}, \nu_{0}\right)$ admits a disintegration with respect to its first marginal $\mu_{0}$ i.e. can be written as $\gamma=\mu_{0} \otimes \gamma^{x}$ where $x \mapsto \gamma^{x}$ is a Borel family of probability measures (naturally interpreted as conditional probability of $y$ given $x$ ). Let us then define for every $x$ :

$$
v_{\gamma}(x):=\inf _{X \in W^{1, q}, X(0)=x} \int_{\mathbb{R}^{d}}\left(\int_{0}^{T} L(X(t), \dot{X}(t), y) d t+\phi(X(T), y)\right) d \gamma^{x}(y) .
$$

Then, the decoupled problem is:

$$
(\mathcal{Q}) \inf _{\gamma \in \Pi\left(\mu_{0}, \nu_{0}\right)} J(\gamma)=\int_{\mathbb{R}^{d}} v_{\gamma}(x) d \mu_{0}(x) .
$$

The novel feature of this decoupled problem is that, contrary to the optimal transport problem $(\mathcal{P})$, it is nonlinear. In fact, since $v_{\gamma}(x)$ is concave in $\gamma^{x}$ (as an infimum of linear functionals of $\gamma^{x}$ ), $(\mathcal{Q})$ is a concave minimization problem over $\Pi\left(\mu_{0}, \nu_{0}\right)$. Existence of a minimizer is a real issue here since the criterion in $(\mathcal{Q})$ may not be weakly lower semicontinuous (in fact even the measurability of $x \mapsto v_{\gamma}(x)$ is not obvious and a counterexample to lower semicontinuity will be given in the next section).

Theorem 2.2. In addition to the previous assumptions, let us assume that $\mu_{0}$ is atomless and that $v(.,$.$) is continuous, then one has$

$$
\inf (\mathcal{Q})=\min (\mathcal{P}) .
$$

Moreover, $\gamma$ solves $(\mathcal{Q})$ if and only if it solves $(\mathcal{P})$ and

$$
v_{\gamma}(x)=\int_{\mathbb{R}^{d}} v(x, y) d \gamma^{x}(y) \mu_{0} \text {-a.e.. }
$$

In particular if $S$ is an optimal transport for $(\mathcal{P})$, i.e. $\mu_{0} \otimes \delta_{S(x)}$ solves $(\mathcal{P})$, then it also solves $(\mathcal{Q})$.

Proof. Since $v_{\gamma}(x) \geq \int_{\mathbb{R}^{d}} v(x, y) d \gamma^{x}(y)$, one immediately deduces that $\inf (\mathcal{Q}) \geq$ $\min (\mathcal{P})$. Now, let us remark that if $\gamma$ is given by a transport map that is $\gamma=\mu_{0} \otimes \delta_{S(x)}$ then $v_{\gamma}(x)=v(x, S(x))$ for $\mu_{0}$-a.e. $x$ and then in this case one has $\int_{\mathbb{R}^{d}} v_{\gamma} d \mu_{0}=\int_{\mathbb{R}^{d}} v(x, S(x)) d \mu_{0}(x)=\int_{\mathbb{R}^{d} \times \mathbb{R}^{d}} v d \gamma$. Since $\mu_{0}$ is atomless the set of transport plans induced by transport maps is weakly-* dense in 
$\Pi\left(\mu_{0}, \nu_{0}\right)$ (see [1]). In particular, there is a minimizing sequence for $(\mathcal{P})$ of the form $\gamma_{n}=\mu_{0} \otimes \delta_{S_{n}(x)}$ where $S_{n} \# \mu_{0}=\nu_{0}$ for every $n$. One then has

$$
\inf (\mathcal{Q}) \leq \int v_{\gamma_{n}} d \mu_{0}=\int v\left(x, S_{n}(x)\right) d \mu_{0}(x)
$$

and passing to the limit one gets $\inf (\mathcal{Q}) \leq \min (\mathcal{P})$. Let $\gamma \in \Pi\left(\mu_{0}, \nu_{0}\right)$, $\gamma$ solves $(\mathcal{Q})$ if and only if

$$
J(\gamma)=\inf (\mathcal{Q})=\min (\mathcal{P}) \geq I(\gamma)
$$

that is $\gamma$ solves $(\mathcal{P})$ and $I(\gamma)=J(\gamma)$ i.e.

$$
v_{\gamma}(x)=\int_{\mathbb{R}^{d}} v(x, y) d \gamma^{x}(y) \mu_{0} \text {-a.e.. }
$$

If we consider again the case

$$
L(x, u, y)=c(x-y)+F(u), \phi(x, y)=\phi(x-y)
$$

under the same convexity, growth and regularity assumptions as in subsection 2.1, then we immediately deduce that if $\mu_{0}$ is absolutely continuous with respect to the Lebesgue measure, then the corresponding decoupled problem admits a unique solution that is given by a transport map.

\section{The linear-quadratic deterministic case}

In this paragraph, we consider the case of a quadratic running cost and zero terminal cost:

$$
c(x, y, u)=\frac{1}{2}|x-y|^{2}+\frac{1}{2}|u|^{2}, \phi(x, y)=0
$$

and we will distinguish the coupled case (where $u$ is allowed to depend on both $x$ and $y$ ) and the decoupled case where $u$ depends on the initial condition $x$ but has to be the same for every $y$. Of course, in this quadratic framework, we will also assume that $\mu_{0}$ and $\nu_{0}$ have finite second-order moments.

The coupled case One first solves for every $(x, y)$ :

$$
v(x, y):=\inf _{X \in H^{1}\left(0, T, \mathbb{R}^{d}\right), X(0)=x} \int_{0}^{T}\left(\frac{1}{2}|X(t)-y|^{2}+\frac{1}{2}|\dot{X}(t)|^{2}\right) d t .
$$


By standard arguments, this problem has unique solution given by:

$$
X(t, x, y):=a(t)(x-y)+y, \quad \text { with } a(t)=\frac{e^{t-2 T}+e^{-t}}{1+e^{-2 T}}, t \in[0, T] .
$$

Replacing in $v$ then leads to the optimal transportation problem:

$$
\frac{1}{2} \int_{0}^{T}\left(a^{2}+\dot{a}^{2}\right) \inf _{\gamma \in \Pi\left(\mu_{0}, \nu_{0}\right)} \int_{\mathbb{R}^{d} \times \mathbb{R}^{d}}|x-y|^{2} d \gamma(x, y)
$$

which is simply the optimal transportation problem with quadratic cost. Denoting by $W^{2}\left(\mu_{0}, \nu_{0}\right)$ the squared-2-Wasserstein distance between $\mu_{0}$ and $\nu_{0}$, we then have the following expression for the value of (3.2):

$$
\frac{1}{2}\|a\|_{H^{1}}^{2} W^{2}\left(\mu_{0}, \nu_{0}\right) \text { with }\|a\|_{H^{1}}^{2}=\frac{1-e^{-2 T}}{1+e^{-2 T}} .
$$

The decoupled case In this case, one has to minimize with respect to $\gamma \in \Pi\left(\mu_{0}, \nu_{0}\right)$ and $(t, x) \mapsto X(t, x)$ (independent of $y$ ) such that $X(0, x)=x$, the quantity

$$
\int_{\mathbb{R}^{d} \times \mathbb{R}^{d}}\left(\int_{0}^{T}\left(\frac{1}{2}|X(t, x)-y|^{2}+\frac{1}{2}|\dot{X}(t, x)|^{2}\right) d t\right) d \gamma(x, y) .
$$

Disintegrating $\gamma$ as $\gamma=\mu_{0} \otimes \gamma^{x}$, we denote by $g_{\gamma}(x)$ the average of $\gamma^{x}$ :

$$
g_{\gamma}(x):=\int_{\mathbb{R}^{d}} y d \gamma^{x}(y) \text {, for } \mu_{0^{-}} \text {a.e. } x \in \mathbb{R}^{d} .
$$

Using the disintegration of $\gamma$ with respect to $\mu_{0}$ and the conditional moment $g_{\gamma}$, it is convenient to rewrite the cost (3.4) as:

$$
\begin{array}{r}
\int_{\mathbb{R}^{d}}\left(\int_{0}^{T}\left(\frac{1}{2}|X(t, x)|^{2}-X(t, x) \cdot g_{\gamma}(x)+\frac{1}{2}|\dot{X}(t, x)|^{2}\right) d t\right) d \mu_{0}(x) \\
+\frac{T}{2} \int_{\mathbb{R}^{d}}|y|^{2} d \nu_{0}(y) .
\end{array}
$$

Minimizing in $X(., x)$ for each fixed $x$ then yields

$$
X(t, x)=a(t)\left(x-g_{\gamma}(x)\right)+g_{\gamma}(x)
$$

where $a$ is as in (3.1). Replacing in (3.4), then yields

$$
v_{\gamma}(x)=\frac{1}{2}\|a\|_{H^{1}}^{2}\left|x-g_{\gamma}(x)\right|^{2}+\frac{T}{2} \int_{\mathbb{R}^{d}}\left|y-g_{\gamma}(x)\right|^{2} d \gamma^{x}(y) .
$$


We thus find that the least cost, as a function of $\gamma \in \Pi\left(\mu_{0}, \nu_{0}\right)$ only, is:

$$
\begin{array}{r}
\frac{1}{2}\|a\|_{H^{1}}^{2} \int_{\mathbb{R}^{d}}|x|^{2} d \mu_{0}(x)+\frac{T}{2} \int_{\mathbb{R}^{d}}|y|^{2} d \nu_{0}(y) \\
+\frac{1}{2}\left(\|a\|_{H^{1}}^{2}-T\right) \int_{\mathbb{R}^{d}}\left|g_{\gamma}\right|^{2} d \mu_{0}-\|a\|_{H^{1}}^{2} \int_{\mathbb{R}^{d}} x \cdot g_{\gamma} d \mu_{0} .
\end{array}
$$

Recalling that $\|a\|_{H^{1}}^{2}$ is given by (3.3), one easily gets $\|a\|_{H^{1}}^{2}-T<0$ for every $T>0$ (which is consistent with the concavity property of the decoupled problem as noticed in section 2) and thus finding an optimal transport plan amounts to:

$$
\inf _{\gamma \in \Pi\left(\mu_{0}, \nu_{0}\right)} J(\gamma)=-A(T) \int_{\mathbb{R}^{d}}\left|g_{\gamma}(x)\right|^{2} d \mu_{0}(x)-2 \int_{\mathbb{R}^{d} \times \mathbb{R}^{d}} x \cdot y d \gamma(x, y)
$$

where $A(T)$ is the positive constant:

$$
A(T):=\frac{T}{\|a\|_{H^{1}}^{2}}-1=\frac{e^{-2 T}(T+1)+T-1}{1-e^{-2 T}} .
$$

Of course when $\mu_{0}$ is absolutely continuous, there is an optimal transport for the quadratic cost and then by theorem 2.2 it is also the unique solution of (3.8). The particular and simple form of the functional $J$ in (3.8) enables us to deduce various counterexamples and some particular simple cases of non existence when $\mu_{0}$ is atomless but may charge small sets.

The functional $J$ is not lower semicontinuous: To see this, let us take $\mu_{0}$ and $\nu_{0}$ equal and uniform on the segment $[-1,1], \gamma_{n}=\mu_{0} \otimes \delta_{S_{n}(x)}$ where $S_{n}$ is a sequence of $\mu_{0}$-measure preserving maps such that $\gamma_{n}$ weakly-* converges to the independent coupling $\mu_{0} \otimes \mu_{0}$, then $\int\left|g_{\gamma}\right|^{2} d \mu_{0}=0$ and then

$$
\limsup _{n} \int\left|g_{\gamma_{n}}\right|^{2} d \mu_{0}=\limsup \left\|S_{n}\right\|_{L^{2}}^{2}>\int\left|g_{\gamma}\right|^{2} d \mu_{0}
$$

since otherwise $S_{n}$ would converge to 0 in $L^{2}$ and then one would have $\gamma=$ $\mu_{0} \otimes \delta_{0}$.

A counterexample to existence: In view of the previous example, one should in general suspect non existence for the minimization of $J$ over $\Pi\left(\mu_{0}, \nu_{0}\right)$. Let us consider the two-dimensional case where $\mu_{0}$ is uniformly distributed on the segment $[0,1] \times\{0\}$ (it thus charges a small set but still is atomless) and let $\nu_{0}$ be uniform on the square $[0,1]^{2}$. Assume that there exists a minimizer $\gamma$ of the decoupled problem. In this case, since $\mu_{0}$ is 
atomless, using theorem 2.2 , the explicit values of $v(x, y)$ and $v_{\gamma}(x)$ given by (3.6), we would then have for $\mu_{0}$-a.e. $x$

$$
\begin{aligned}
v_{\gamma}(x) & =\frac{1}{2}\|a\|_{H^{1}}^{2}\left|x-g_{\gamma}(x)\right|^{2}+\frac{T}{2} \int_{\mathbb{R}^{d}}\left|y-g_{\gamma}(x)\right|^{2} d \gamma^{x}(y) \\
& =\int_{\mathbb{R}^{d}} v(x, y) d \gamma^{x}(y)=\frac{1}{2}\|a\|_{H^{1}}^{2} \int_{\mathbb{R}^{d}}|x-y|^{2} d \gamma^{x}(y) \\
& \left.=\left.\frac{1}{2}\|a\|_{H^{1}}^{2}\left(\left|x-g_{\gamma}(x)\right|^{2}+\int_{\mathbb{R}^{d}} \mid y-g_{\gamma}(x)\right)\right|^{2} d \gamma^{x}(y)\right) .
\end{aligned}
$$

Which implies that $\gamma^{x}$ should be a Dirac mass for $\mu_{0}$-a.e. $x$, it would thus imply that $\gamma$ is induced by a transport map $S=\left(S_{1}, S_{2}\right)$. At this stage it is not possible to conclude directly since there exist measure preserving maps between $\mu_{0}$ and $\nu_{0}$, and even continuous ones such as Peano's function (see for instance [10]). To derive the desired contradiction, one has to use further theorem 2.2 to deduce that $S$ is also an optimal transport map between $\mu_{0}$ and $\nu_{0}$ for the quadratic cost. Thus, by well-known arguments (see [8], [11], [12]) there exists a convex function $(x, y) \in[0,1]^{2} \mapsto \psi(x, y)$ such that $\left(S_{1}(x, 0), S_{2}(x, 0)\right) \in \partial \psi(x, 0)$ for (Lebesgue)-a.e. $x \in[0,1]$ which easily implies that $S_{1}(., 0)$ is nondecreasing and since $S_{1}(., 0)$ preserves the Lebesgue measure this yields $S_{1}(x, 0)=x$. Now since $S(x, 0)=\left(x, S_{2}(x, 0)\right)$ is Borel and measure-preserving between $\mu_{0}$ and $\nu_{0}$ we should have

$$
\int_{0}^{1} \varphi\left(x, S_{2}(x, 0)\right) d x=\int_{[0,1]^{2}} \varphi(x, y) d x d y
$$

for every Borel and bounded $\varphi:[0,1]^{2} \rightarrow \mathbb{R}$, so that taking $\varphi(x, y)=$ $\left(y-S_{2}(x, 0)\right)^{2}$ yields the desired contradiction.

The discrete case, different values for the coupled and decoupled problems: Let us now consider the case where both $\mu_{0}$ and $\nu_{0}$ have finite support, in this case (3.8) is in fact a finite-dimensional problem and it is easy to check that it possesses solutions. However, because of atoms, theorem 2.2 does not apply and it is easy to find a case where the values are different. To see this, let us choose $d=1, \mu_{0}=\delta_{0}$ and $\nu_{0}=\frac{1}{2} \delta_{-1}+\frac{1}{2} \delta_{1}$. The set of transport plans reduces to the single element $\gamma=\frac{1}{2} \delta_{(0,1)}+\frac{1}{2} \delta_{(0,-1)}$. Obviously, the transport plan $\gamma$ is not induced by any transport map. In this example, the solution of both the coupled and the decoupled problem is the transport plan $\gamma$. A direct computation leads to $\inf (\mathcal{Q})-\inf (\mathcal{P})=\frac{1}{2}\left(T-\|a\|_{H^{1}}^{2}\right)>0$. 


\section{References}

[1] L. Ambrosio, Lecture notes on optimal transport problems, Lecture Notes in Math., 1812, Springer, Berlin, (2003).

[2] Y. Brenier, Polar factorization and monotone rearrangement of vectorvalued functions, Comm. Pure Appl. Math., 44, 375-417 (1991).

[3] P.A. Chiappori, R.J. Mc Cann, L. Nesheim. Hedonic price equilibria, stable matching, and optimal transport: equivalence, topology, and uniqueness, to appear in Econom. Theory.

[4] I. Ekeland, An optimal matching problem, ESAIM Control Optim. Calc. Var., 11, no. 1, 57-71(2005).

[5] I. Ekeland, Existence, uniqueness and efficiency of equilibrium in hedonic markets with multidimensional types, to appear in Econom. Theory.

[6] W. H. Fleming, H.M. Soner, Controlled Markov Processes and Viscosity Solutions, Graduate Studies in Mathematics, 58, Springer-Verlag, Applications of Mathematics, (1993).

[7] W. Gangbo, An elementary proof of the polar factorization of vectorvalued functions, Arch. Rational Mech. Anal., 128, 380-399 (1994).

[8] W. Gangbo, R.J. McCann, The Geometry of Optimal Transportation, Acta Math., 177 , 113-161 (1996).

[9] R.J. McCann, Existence and uniqueness of monotone measurepreserving maps, Duke Math. J., 80, 309-323 (1995).

[10] A.H. Stone, Measure-preserving maps in General topology and its relations to modern analysis and algebra, IV (Proc. Fourth Prague Topological Sympos., Prague, 1976), Part A, , Lecture Notes in Math., 609, Springer, Berlin, pp. 205-210 1977.

[11] C. Villani, Topics in optimal transportation, Graduate Studies in Mathematics, 58, American Mathematical Society, Providence, RI, (2003).

[12] C. Villani, Optimal transport. Old and new, Springer-Verlag, Berlin, (2009). 